\title{
Endocrine Surgery in the Coronavirus Disease 2019 Pandemic
}

\author{
Yelda Jozaghi et al. ${ }^{1}$ \\ ${ }^{1}$ Affiliation not available
}

April 28, 2020

\begin{abstract}
Background

In the face of the COVID-19 pandemic, cancer care has had to adapt rapidly given the Centers for Disease Control and Prevention (CDC) and the American College of Surgeons (ACS) issuing recommendations to postpone non-urgent surgeries.

Methods

An institutional multidisciplinary group of Head and Neck Surgical Oncology, Surgical Endocrinology, and Medical Endocrinology devised Surgical Triaging Guidelines for Endocrine Surgery during COVID-19, aligned with phases of care published by the ACS.

\section{Results}

Phases of care with examples of corresponding endocrine cases are outlined. Most cases can be safely postponed with active surveillance, including most differentiated and medullary thyroid cancers. During the most acute phase, all endocrine surgeries are deferred except thyroid tumors requiring acute airway management.
\end{abstract}

\section{Conclusions}

These guidelines provide context for endocrine surgery within the spectrum of surgical oncology, with the goal of optimal individualized multidisciplinary patient care, and the expectation of significant resource diversion to care for COVID-19 patients.

\section{Full author list}

Yelda Jozaghi*1, Mark E Zafereo*1, Nancy D Perrier ${ }^{2}$, Jennifer R Wang ${ }^{1}$, Elizabeth Grubbs ${ }^{2}$, Neil D Gross ${ }^{1}$, Sarah Fisher ${ }^{2}$, Erich M Sturgis ${ }^{1}$, Ryan P Goepfert ${ }^{1}$, Stephen Y Lai ${ }^{1}$, Conor Best ${ }^{3}$, Naifa L Busaidy ${ }^{3}$, Maria E Cabanillas ${ }^{3}$, Ramona Dadu ${ }^{3}$, Robert F Gagel ${ }^{3}$, Mouhammed A Habra ${ }^{3}$, Mimi I Hu ${ }^{3}$, Camilo Jimenez ${ }^{3}$, Steven I Sherman ${ }^{3}$, Sonali Thosani ${ }^{3}$, Jeena Varghese ${ }^{3}$, Steven G Waguespack ${ }^{3}$, Steven Weitzman ${ }^{3}$, Anita K Ying ${ }^{3}$, Paul H Graham ${ }^{2}$. ${ }^{*}$ Co-first authors

${ }^{1}$ Department of Head and Neck Surgery, The University of Texas MD Anderson Cancer Center, Houston, TX, USA. ${ }^{2}$ Department of Surgical Oncology, The University of Texas MD Anderson Cancer Center, Houston, TX, USA. ${ }^{3}$ Department of Endocrine Neoplasia and Hormonal Disorders, The University of Texas MD Anderson Cancer Center, Houston, TX, USA.

This work was not funded.

All authors participated in the manuscript drafting or review process.

This work has not been presented at any meetings or conferences.

Corresponding author: Mark E Zafereo 1515 Holcombe Boulevard Unit FCT 10.6008 Houston, Texas 77030 713-563-9683 MZafereo@mdanderson.org

Key words: Coronavirus, COVID, Endocrine, Surgery, Guideline. 
Facing the realities of the coronavirus disease 2019 (COVID-19) pandemic, governments around the world and in the United States have implemented societal interventions such as "social distancing" measures, "stay at home" orders, border closures, and nationwide lockdowns. The underlying goal is to curtail all non-essential population movement in an attempt to limit the rate of spread of this virus. Similar measures have been undertaken in the medical community to prioritize patient encounters to those necessitating timely evaluation and treatment.

Several populations are considered to be at higher risk for complications of a COVID-19 infection. These include immunocompromised patients such as those undergoing active or recent oncologic treatment. Mortality rates among cancer patients have been reported to be $29 \%^{1}$. In a nationwide analysis of COVID-19 cases in China, cancer patients were noted to have a $39 \%$ rate of severe events as compared with $8 \%$ in the non-cancer population. These severe events included ICU admissions requiring ventilation or death ${ }^{2}$.

Cancer care has had to adapt rapidly to the evolving pandemic and accommodate the particular vulnerability of the oncologic patient population to COVID-19. In March 2020, both the Centers for Disease Control and Prevention (CDC) and the American College of Surgeons (ACS) issued recommendations to postpone or cancel elective surgeries ${ }^{3}$. Increasingly, hospitals have been forced to reallocate resources to the care of the critically ill. The ACS later expanded on their recommendations to triage various oncologic surgeries based on each hospital's COVID-19 preponderance and availability of local resources, which they divide into 3 phases of care $^{4,5}$. The National Comprehensive Cancer Network has highlighted the unique challenges of cancer care during this time and has encouraged the coordination of organizational structures to facilitate collaborative, thoughtful care of cancer patients ${ }^{6}$.

Several large subspecialty societies have recently published surgical guidelines for the care of patients with endocrine tumors during the COVID-19 pandemic ${ }^{7-10}$. In addition, many medical centers and academic institutions have developed their own clinical practice guidelines to triage oncologic patient care. In preparation for the pandemic, The University of Texas MD Anderson Cancer Center has established multidisciplinary endocrine surgery guidelines and initiated specific conferences to determine case urgency. As a multidisciplinary group consisting of Head and Neck Surgical Oncology, Surgical Endocrinology, and Medical Endocrinology, we have devised the following Surgical Triaging Guidelines for Endocrine Surgery at the time of a pandemic such as COVID-19. These guidelines apply specifically to surgical procedures. Other adjuvant therapies, particularly postoperative radioactive iodine ablation, may also be safely deferred in the context of this pandemic.

We have aligned the case priorities to the phases of care outlined by the ACS and have triaged case acuity based on the American College of Surgeons Elective Surgery Acuity Scale ${ }^{11}$. Phases of care with added examples of corresponding endocrine cases have been provided. According to the listed acuity descriptions, most cases (including the majority of differentiated thyroid carcinoma) can be safely postponed with active surveillance. These guidelines provide a context for endocrine surgery within the entire spectrum of surgical oncology, especially when overall priorities may focus on ensuring available Personal Protective Equipment (PPE), ICU resources, and adequate protection of medical personnel during the COVID-19 pandemic. While these recommendations are intended to serve as general principles, and we continue to advocate that where possible surgeries be considered individually in a multidisciplinary setting based on the unique patient and environment circumstances.

\section{Proposed Endocrine Surgery Triaging Algorithm Aligned with the American College of Sur- geons Phases of Care}

Phase I.Few COVID-19 patients, hospital resources not exhausted, institution still has ICU ventilator capacity and COVID-19 trajectory not in rapid escalation phase.

Surgery restricted to patients likely to have survivorship compromised if surgery not performed within next 3 months. 


\section{Surgical cases that need to be performed as soon as feasible:}

- Thyroid cancer requiring acute airway management

- Resectable anaplastic or poorly differentiated thyroid cancer withoutBRAFV600E mutation*

- Progressive/clinically aggressive differentiated or medullary thyroid cancer

- Large suspected thyroid malignancy with documented progression

- Large goiters with significant symptomatic airway compression**

- Suspected parathyroid carcinoma with significant symptomatic hypercalcemia

- Suspected adrenocortical carcinoma

- Medically-uncontrolled hyperfunctioning endocrine tumors

Surgical cases that can be safely deferred:

- Differentiated thyroid cancer***

- Medullary thyroid cancer***

- Indeterminate thyroid nodules without documented progression

- Thyroid goiters**

- Primary hyperparathyroidism

- Medically-controlled hyperfunctioning endocrine tumors

- Incidental, nonfunctional adrenal lesions

*Anaplastic thyroid cancer patients with BRAFV600E mutations should be offered BRAF/MEK inhibitor therapy initially and surgery can be deferred ${ }^{12}$.

**Generally, mildly symptomatic airway compression from large goiters is subacute or chronic, and does not require immediate intervention. Very rarely, a patient may present with significant and acute airway compromise, in which case surgery or airway intervention cannot be safely postponed.

***Most differentiated and medullary thyroid cancers are slow-growing tumors that don't cause significant morbidity over the short-term, although there are a subset of more biologically aggressive cancers that progress more rapidly. Therefore, clinical correlation with rate of progression (if known), size, invasiveness of surrounding structures, and proximity to critical structures is recommended.

Phase II. Many COVID-19 patients, ICU and ventilator capacity limited, and/or Operating Room supplies limited.

Surgery restricted to patients likely to have survivorship compromised if surgery not performed within next few days.

\section{Surgical cases that need to be performed as soon as feasible:}

- Thyroid cancers requiring acute airway management

- Resectable anaplastic or rapidly progressive poorly differentiated thyroid cancer without BRAFV600E mutation ***

- Rapidly progressive/clinically aggressive differentiated or medullary thyroid cancers***

- Rapidly progressive adrenocortical carcinoma

- Medically-uncontrolled hyperfunctioning endocrine tumors

- Suspected parathyroid cancer with significant hypercalcemia refractory to medical management

\section{All other endocrine diagnoses can generally be deferred.}

***Some anaplastic, poorly differentiated, and advanced progressive differentiated and medullary cancers require major surgery such as laryngectomy, tracheal resection, and/or sternotomy. These more advanced and complex cases should be considered in alignment with available hospital resources.

Phase III. Hospital resources are all routed to COVID 19 patients, no ventilator or ICU capacity, OR supplies exhausted. 
Surgery restricted to patients likely to have survivorship compromised if surgery not performed within next few hours.

\section{Surgical cases that need to be performed as soon as feasible:}

Thyroid cancer requiring acute airway management

\section{All other endocrine diagnoses are deferred.}

\section{Alternative treatment approaches for endocrine neoplasia for whom surgical intervention is deferred due to COVID-19 pandemic:}

- Most surgeries for thyroid nodules, thyroid goiters, differentiated thyroid cancers, and medullary thyroid cancers and adrenal nodules can be safely deferred without need for medical therapy

- Consider TSH suppression for differentiated thyroid cancers

- Anaplastic, poorly differentiated, or progressive/advanced differentiated and medullary thyroid cancers with targetable mutations may be candidates for targeted systemic therapy

- Optimize medical management of hyperfunctioning endocrine tumors (thyroid, parathyroid, and adrenal)

- Coordination of care with other health-care providers/facilities according to resource availability and travel restrictions

\section{References}

1. Zhang L, Zhu F, Xie L, Wang C, et al. Clinical characteristics of COVID-19-infected cancer patients: A retrospective case study in three hospitals within Wuhan, China. Ann Oncol. 2020 Mar 26.

2. Liang W, Guan W, Chen R, et al. Cancer patients in SARS-CoV-2 infection: a nationwide analysis in China. Lancet Oncol. 2020 Mar;21(3):335-337.

3. Sealander KS, Dombey D, Rogers A, Miller M, Madhani S. How to Handle Elective Surgeries and Procedures During the COVID-19 Pandemic. Published online March 2, 2020. Accessed online April 1, 2020. https://www.mwe.com/insights/how-to-handle-elective-surgeries-and-procedures-during-the-covid19-pandemic/

4. American College of Surgeons, Commission on Cancer. COVID-19 Guidelines for Triage of Colorectal Cancer Patients. American College of Surgeons. Published online March 24, 2020. Accessed online April 1, 2020. https://www.facs.org/covid-19/clinical-guidance/elective-case/colorectal-cancer

5. American College of Surgeons, Commission on Cancer. COVID-19 Guidelines for Triage of Thoracic Patients. American College of Surgeons. Published online March 24, 2020. Accessed online April 1, 2020. https://www.facs.org/covid-19/clinical-guidance/elective-case/thoracic-cancer

6. Ueda M, Martins R, Hendrie PC, et al. Managing Cancer Care During the COVID-19 Pandemic: Agility and Collaboration Toward a Common Goal. J Natl Compr Canc Netw. 2020 Mar 20.

7. Stack B, Randolph G. Endocrine Surgery during the Covid-19 pandemic. American Head and Neck Society Published online March 24, 2020. Accessed online April 3, 2020. https://www.ahns.info/wpcontent/uploads/2020/03/Endocrine-Surgery-during-the-Covid.pdf

8. Givi B, Schiff BA, Chinn SB, et al. Safety Recommendations for Evaluation and Surgery of the Head and Neck During the COVID-19 Pandemic. JAMA Otolaryngol Head Neck Surg. 2020 Mar 31.

9. Society of Surgical Oncology. Resource for Management Options of Endocrine/Head and Neck Cancer During COVID-19. Published online March 30, 2020. Accessed online April 3, 2020. https://www.surgonc.org/wpcontent/uploads/2020/03/Endocrine-Head-and-Neck-Resource-during-COVID-19-3.30.20.pdf

10. American Association of Endocrine Surgeons. Accessed online April 3, 2020. https://www.endocrinesurgery.org/ 
11. American College of Surgeons. COVID-19: Guidance for Triage of Non-Emergent Surgical Procedures. Published online March 17, 2020. Accessed online April 1, 2020. https://www.facs.org/covid-19/clinicalguidance/triage

12. Wang JR, Zafereo ME, Dadu R, et al. Complete Surgical Resection Following Neoadjuvant Dabrafenib Plus Trametinib in BRAFV600E-Mutated Anaplastic Thyroid Carcinoma. Thyroid. 2019 Aug;29(8):10361043. 